\title{
Trigeminal ganglion transcriptome analysis in 2 rat models of medication-overuse headache reveals coherent and widespread induction of pronociceptive gene expression patterns
}

Daniela Buonvicino ${ }^{a}$, Matteo Urru ${ }^{a}$, Mirko Muzzi ${ }^{a}$, Giuseppe Ranieri ${ }^{a}$, Cristina Luceri ${ }^{b}$, Claudia Oteri $^{a}$, Andrea Lapucci ${ }^{a}$, Alberto Chiarugi $i^{a, \star}$

\begin{abstract}
We attempted to gather information on the pathogenesis of medication-overuse headache, as well as on the neurochemical mechanisms through which symptomatic medication overuse concurs to headache chronification. Transcriptional profiles were therefore evaluated as an index of the homeostasis of the trigeminovascular system in the trigeminal ganglion of female rats exposed for 1 month to daily oral doses of eletriptan or indomethacin. We report that both drug treatments change trigeminal ganglion gene expression to a similar extend. Of note, qualitative transcriptomic analysis shows that eletriptan and indomethacin prompt nearly identical, increased expression of genes coding for proteins involved in migraine pathogenesis and central pain sensitization such as neuropeptides, their cognate receptors, prostanoid, and nitric oxide-synthesizing enzymes, as well as TRP channels. These genes, however, were not affected in thoracic dorsal root ganglia. Of note, lowering of orofacial nociceptive thresholds, as well as forepaw hyperalgesia occurred in both indomethacin- and eletriptan-treated rats. Our study reveals that chronic rat exposure to 2 acute headache medications with completely different mechanisms of action prompts pain sensitization with highly similar induction of pronociceptive genes selectively within the trigeminal ganglion. Data further our understanding of medication-overuse headache pathogenesis and provide hints for specific mechanism-based treatment options.
\end{abstract}

Keywords: Medication-overuse headache, Eletriptan, Indomethacin, Nonsteroidal anti-inflammatory drugs

\section{Introduction}

Medication-overuse headache $(\mathrm{MOH})$ is a debilitating, chronic headache with a 1-year prevalence of $1 \%$ to $2 \% .{ }^{13,21}$ Whether $\mathrm{MOH}$ is a biological entity that differs from chronic daily headache and shows specific neurobiological and genetic background is a matter of debate. ${ }^{23}$ Although $\mathrm{MOH}$ tends to show the same features regardless of the overused drug, a longitudinal study in migraineurs suggests that the 1-year probability of migraine transformation into $\mathrm{MOH}$ is higher for barbiturates and progressively decreases for opioids, triptans, and nonsteroidal anti-inflammatory drugs (NSAIDs). ${ }^{5}$ The temporal kinetics characterizing the shift from correct symptomatic therapy to regular (often daily) overuse also depend on headache severity, age, sex, and psychological status of the patient. ${ }^{5,6,10}$ Clinically, $\mathrm{MOH}$ represents a critical condition where a vicious cycle of chronic headache and regular symptomatic medication intake is

Sponsorships or competing interests that may be relevant to content are disclosed at the end of this article.

a Department of Health Sciences, Section of Clinical Pharmacology and Oncology, University of Florence, Florence, Italy, ${ }^{b}$ Department of NEUROFARBA, Division of Pharmacology and Toxicology, University of Florence, Florence, Italy

${ }^{*}$ Corresponding author. Address: Department of Health Sciences, Section of Clinical Pharmacology and Oncology, University of Florence, Viale Pieraccini 6 50139 Florence, Italy. Tel.: +39-055-4271230; fax: +39-055-4271280. E-mail address: alberto.chiarugi@unifi.it (A. Chiarugi).

PAIN 159 (2018) 1980-1988

(C) 2018 International Association for the Study of Pain

http://dx.doi.org/10.1097/j.pain.0000000000001291 supposed to underlie drug overuse. ${ }^{16}$ Patients with $\mathrm{MOH}$ are typically refractory to classic preventive treatments, and there is general agreement that withdrawal from medication overuse is mandatory to reestablish previous headache features, as well as sensitivity to headache prophylaxis. ${ }^{2,30,36}$ Overall, $\mathrm{MOH}$ represents a problem of social health with important therapeutic, toxicological, and pharmacoeconomic implications. ${ }^{15,33}$

A great deal of efforts is currently directed at understanding the molecular and pathophysiological basis that underlies $\mathrm{MOH}$ pathogenesis. ${ }^{14}$ At present, 2 nonmutually exclusive hypotheses have been put forward. ${ }^{6}$ The first is based on the compulsive use of headache medications shown in some patients with $\mathrm{MOH}$ and considers $\mathrm{MOH}$ as an addiction to symptomatic remedies. ${ }^{22,35}$ According to this interpretation, therefore, dysfunction of classic reward-seeking central nervous system pathways and drug dependence lays at the basis of $\mathrm{MOH} .{ }^{9}$ A second hypothesis is focused on neural sensitization. The latter occurs as a direct consequence of drug overuse that prompts adaptations of the trigeminovascular system that translates into pain transmission facilitation and latent sensitization. ${ }^{17,28}$ In keeping with this interpretation, work done in rodent models of $\mathrm{MOH}$ suggests that upregulation of calcitonin gene-related peptide (CGRP) and nitric oxide (NO) signaling is causative to the processes of sensitization by triptan overuse. ${ }^{18-20,24}$ However, it is still unknown whether these effects are also shared by symptomatic headache medications different from triptans, and represent a signature of the neurophysiologic rearrangements that underlie $\mathrm{MOH}$. Also, the quantitative aspects, in terms of number of neurotransmitter/neuromodulators involved, that characterize 
the trigeminovascular alterations prompted by repetitive exposure to acute headache remedies still wait to be defined. Addressing these issues might further our understanding of the pathophysiology of $\mathrm{MOH}$ and headache chronification.

Thus, to gather further information on the neurochemistry of $\mathrm{MOH}$, in this study, we designed experiments aimed at understanding whether chronic rat exposure to triptan or NSAID affects transcriptome of the trigeminal and thoracic dorsal root ganglia (DRG), and whether these changes were coherent between the 2 drug classes.

\section{Materials and methods}

\subsection{Animals}

Adult female Wistar rats 230 to $250 \mathrm{~g}$ (Charles River, Milan, Italy) were maintained 3 per cage in a climate-controlled room at $23 \pm$ $2^{\circ} \mathrm{C}$ on a 12-hour light/dark cycles with free access to food and water. All animal manipulations were performed according to the European Community guidelines for animal care (DL 116/92, application of the European Communities Council Directive 86/ 609/EEC).

\subsection{Drug administration}

Rats were daily treated by oral gavage for 30 days with $0.8 \mathrm{mg} / \mathrm{kg}$ eletriptan (ELE) and $2 \mathrm{mg} / \mathrm{kg}$ indomethacin (INDO) dissolved in $100 \mu \mathrm{L}$ of water. Control animals received the same volume of water. Both drugs were from Sigma-Aldrich (Milan, Italy). At the end of the treatments, rats were anesthetized with isoflurane and killed for tissue extraction. The trigeminal ganglion or thoracic DRG were removed and frozen in Trizol at $-80^{\circ} \mathrm{C}$ for RNA extraction.

\subsection{Microarray-based gene expression profiling}

The labeling and hybridization steps were performed according to the Agilent 2-Color Microarray-Based Gene Expression Analysis version 5.7 protocol. Pools of RNA from ganglia of ELE- or INDOtreated rats were contrasted with reference RNA obtained by pooling equal amount of RNAs from control animals, on Whole rat genome Agilent $8 \times 60 \mathrm{~K}$ arrays. Image analysis and quality control were performed as previously reported. ${ }^{27}$

Data were clustered in groups of genes that behaved similarly across the experiments using Cluster 3.0 and Treeview software. Pathway analysis was performed by means of the GO-Elite software, version 1.2 using as input data, the list of the differentially expressed genes from every comparison, with a threshold at 3 and a statistical significance $<0.05$.

The microarray data sets supporting the results of this article are available in the MIAME public database ArrayExpress repository (http://www.ebi.ac.uk/arrayexpress/) (Accession Number E-MTAB-5864).

\subsection{Reverse transcript and real-time polymerase chain reaction}

Total RNA from the trigeminal ganglia or thoracic DRG was isolated using Trizol Reagent (Life Technologies, Monza, Italy). Reverse transcript and quantitative polymerase chain reaction (qPCR) were performed as previously reported. ${ }^{8}$ The following primers were used: for VIP forward 5'-CAGTTCCTGGCGATCCTGACA-3' and reverse 5'-GGGCGTGTCATTCTCCGCTAAGG-3', PACAP forward 5'-TCAGCTTCCCTGGGATCAGACC-3' and reverse
5'-GGTCCAAGACTTTGCGGTAGGCTT-3'， CGRP $\alpha$ forward 5'-GGCATGCGGCCTCCAGGCAG-3' and reverse 5'-AGAGCCCTCAGCCTCCTGTTCCT-3', SST forward 5'-AAGTCTCTGGCGGCTGCCACC-3' and reverse 5'-GCAGCCAGCTTTGCGTTCCCG-3', TRPV1 forward 5'-GCCATGCTCAATCTGCACATTGGG-3' and reverse 5'-GCTCCATTCTCCACCAAGAGG-3', 5HT1RB forward 5'-CATCTCTGTGTCATCGCCCTGGAC-3' and reverse 5'CGTGGTCGGTGTTCACAAAGCAGT-3', 5HT1RD forward 5'-CGAGGAGATGTCTGACTGCCTGGT-3' and reverse 5'CAGAGCCCGTCATAAGCTGTGCTG-3', COX1 forward 5'-GGCCTCGACCACTACCAATGTGAC-3' and reverse 5'AGTACCAGGCGCATGAGTACTTCTCG-3', COX2 forward 5'-GCCGGGTCTGATGATGTATGC-3' and reverse 5'-TGGAACAGTCGCTCGTCATCC-3', and 18S5'-GGGGAATCAGGGTTCGATTCCGG-3' and reverse 5'-GGCACCAGACTTGCCCTCCAATGG-3' . Ten rats per group were used for $\mathrm{QPCR}$ analysis.

\subsection{Behavioral tests}

All behavioral tests were conducted by an experimenter blind to treatment. On each testing day, the animals were brought into the behavior room 1 hour before the test session. Plantar or orofacial allodynia were measured as previously reported. ${ }^{12,32,34}$ Evaluation of orofacial hyperalgesia was conducted by supraorbital injection of a low concentration of formalin $(0.2 \%, 20 \mu \mathrm{L})$, and time spent scratching the injected region consisting of an early short-lasting period (0-10 minutes) followed by a second tonic phase (10-60 minutes) was monitored with a manual chronometer and considered as indicative of nociception. ${ }^{31}$ Grimace score test was conducted as reported. ${ }^{25}$ Cold sensitivity was assessed with the hot/cold-plate analgesiometer (Ugo Basile, Milan, Italy). Animals were allowed to acclimate to the testing apparatus for 30 minutes, after which they were individually placed on the center of a cold plate in a transparent Plexiglas cylinder. The temperature of the cold plate was kept constant at 5 $\pm 1^{\circ} \mathrm{C}$. Pain-related behaviors (ie, lifting and licking of the paw) were observed, and the time(s) of the first sign was recorded. Each trial was repeated 3 times at 10-minute intervals, and a cutoff time of 300 seconds was used to prevent tissue damage. Von Frey experiments were conducted at day 30 , whereas the formalin test was conducted at day 31 in the same animals. In a separate group of animals, the cold plate test and CGRP plasma levels were evaluated at day 31. Behavioral testing occurred before drug administration. Capsaicin was injected only once for a single evaluation of CGRP blood content 1 hour after the cold plate test.

\subsection{Calcitonin gene-related peptide plasma levels}

One hour later the cold plate test, rats received a single injection of $20 \mu \mathrm{L}$ of $0.2 \%$ capsaicin (Sigma Chemical Co, St. Louis, MO) into the vibrissa pad by means of subcutaneous injection with a 30gauge needle. Ten minutes later, blood samples were collected in heparinized Eppendorf tubes by direct puncture of the retroorbital venous plexus. Calcitonin gene-related peptide plasma concentrations were determined by means of an ELISA kit (Bertin Pharma, Montigny le Bretonneux, France).

\subsection{Data analysis}

All numerical data are expressed as mean \pm SEM. For each single gene, differences in expression levels were statistically evaluated comparing treated and untreated conditions using analysis of variance plus the Tukey post hoc test. 

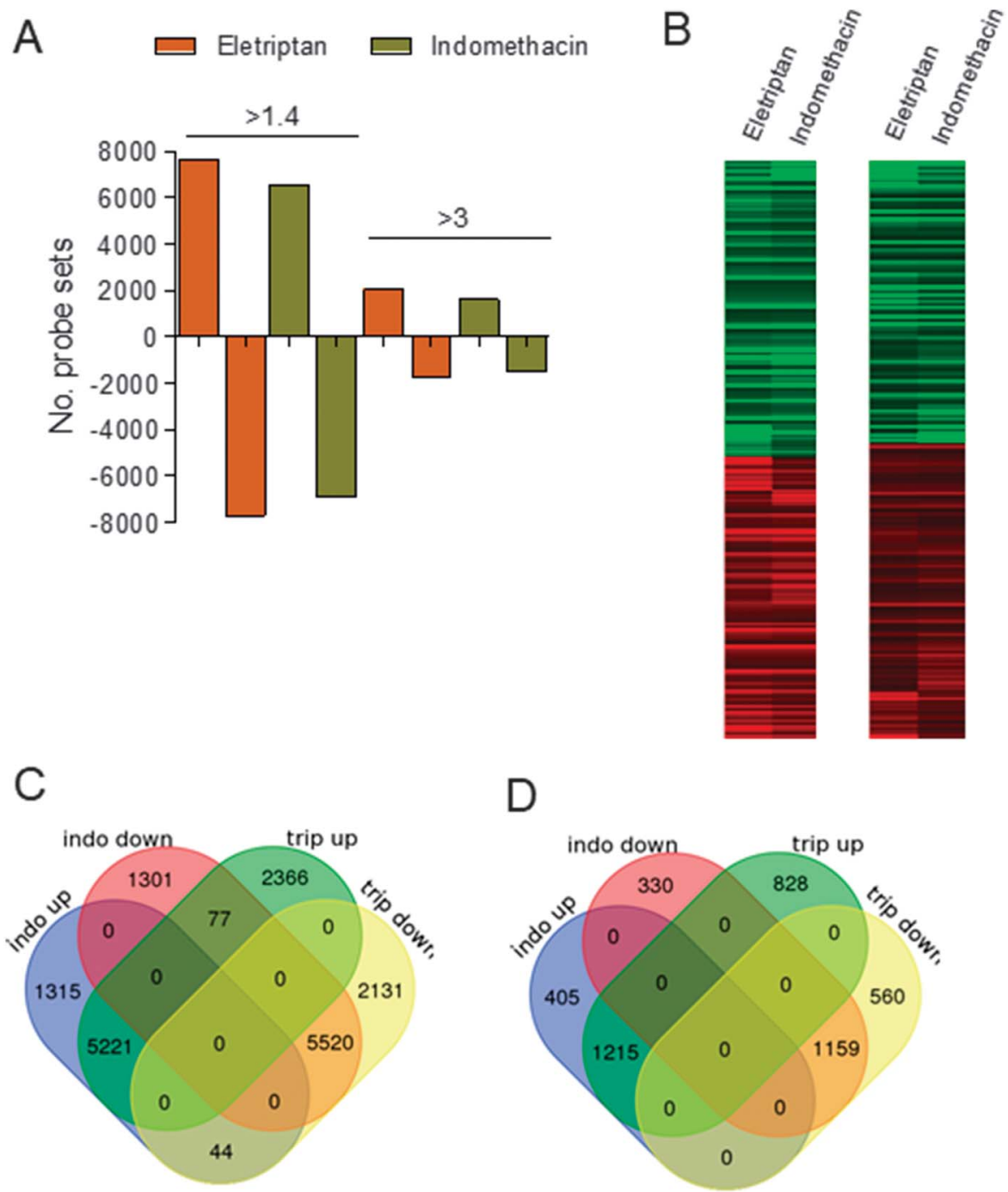

Figure 1. Gene array transcriptomic analysis in the trigeminal ganglion of rats exposed to chronic treatment with ELE or INDO. (A) The number of genes found increased or decreased by 1.4- or 3-fold by means of gene array transcriptomic analysis in the trigeminal ganglion of rats exposed to 1-month daily oral treatment with ELE $(0.8 \mathrm{mg} / \mathrm{kg})$ or INDO (2 mg/kg) compared with control animals as shown. Note the similar changes induced by the 2 different treatments. (B) Hierarchical cluster analysis performed on trigeminal ganglion genes showing a fold change of at least 1.4 (left) or 3 (right) between treated (ELE or INDO) and control animals. Red, upregulated genes; green, downregulated genes. Note the correspondence between genes upregulated or downregulated in ELE- and INDO-exposed rats. Venn diagram showing the number of genes upregulated or downregulated, as well as the degree of their overlapping, in the trigeminal ganglion of rats exposed to ELE or INDO adopting a threshold of 1.4 (C) or 3 (D). ELE, eletriptan; INDO, indomethacin.

\section{Results}

\subsection{Transcriptomic analysis in trigeminal ganglia of rats chronically exposed to ELE or INDO}

To establish a model of $\mathrm{MOH}$, we treated adult female rats with daily oral doses of ELE or INDO for 1 month. ELE was chosen because it represents an efficacious and widely used triptan, whereas INDO was selected among the different NSAIDs because it is one of the most commonly used antimigraine drugs and frequently overused by patients with $\mathrm{MOH}$ in single or combined formulations. We adopted doses of $0.8 \mathrm{mg} / \mathrm{kg}$ for ELE and $2 \mathrm{mg} / \mathrm{kg}$ for INDO in rats that in patients approximately reproduce an overuse of $60 \mathrm{mg} /$ day of ELE (between 1 and 2 tablets of $40 \mathrm{mg}$ ) and $140 \mathrm{mg} /$ day of INDO (between 1 and 2 tablets of $100 \mathrm{mg} /$ day).

The possible impact of both drugs on gene expression profile in the rat trigeminal ganglion was evaluated by means of gene-array analysis. After quality control of the 30,367 probe sets present on the array, 27,297 and 28,393 genes were considered expressed for ELE and INDO, respectively. To understand the degree of consistency of the changes induced by ELE and INDO, we compared the quantitative and qualitative aspects of the results obtained using a fold change threshold of 1.4 and 3 . As shown in Figure 1A, we found that with both thresholds the number of genes whose expression was increased or decreased by INDO and ELE was highly similar. Specifically, with the threshold set at 1.4, the number of induced genes was 7664 (28.1\%) and 6580 (23.2\%) with ELE and INDO, respectively. When the threshold was raised to 3 , induced genes were $2043(7.5 \%)$ and 1621 (5.7\%) for ELE and INDO, respectively (Fig. 1A). Similarly, the number of genes whose expression was downregulated by 1.4 -fold was 7695 (28.2\%) with ELE and 6898 (24.3\%) with INDO, whereas with the threshold set at 3 the number of downregulated genes was 1719 (6.3\%) and 1489 (5.2\%) for ELE and INDO, respectively (Fig. 1A). 


\section{Table 1}

Gene pathways downregulated or upregulated in the trigeminal ganglion of rats chronically exposed to ELE.

\begin{tabular}{|c|c|c|c|c|}
\hline Gene-set name & No. of altered genes & Total no. of genes & $P$ & Gene symbols \\
\hline \multicolumn{5}{|l|}{ Pathways downregulated } \\
\hline Cell cycle: WP429 & 18 & 74 & 0.001 & $\begin{array}{l}\text { Bub1|Bub1b|Ccna2|Ccnb1|Ccne2|Cdc6|Cdk1| } \\
\text { Cdk4|Cdk6|Cdkn2a|Espl1|Hdac7|Mad2|1|Mcm2| } \\
\text { Mcm4|Mcm5|Mcm6|Pkmyt1 }\end{array}$ \\
\hline G1 to S cell cycle control: WP348 & 14 & 58 & 0 & $\begin{array}{l}\text { Ccnb1ICcne2ICdk1ICdk4|Cdk6ICdkn2alCreb3|1| } \\
\text { Mcm2IMcm4IMcm5IMcm6IMyc|Myt1|Pole2 }\end{array}$ \\
\hline Hedgehog signaling pathway: WP574 & 5 & 17 & 0.014 & Ccnb1|Cdk1|Gli2||hh|Stk36 \\
\hline Nuclear receptors: WP217 & 7 & 36 & 0.014 & ArlEsr1|Nr0b1|Nr5a1|Pgr|RorclThrb \\
\hline DNA replication: WP484 & 7 & 37 & 0.019 & Cdc6IMcm10|Mcm2IMcm4|Mcm5|Mcm6|Pole2 \\
\hline \multicolumn{5}{|l|}{ Pathways upregulated } \\
\hline $\begin{array}{l}\text { Prostaglandin synthesis and regulation: } \\
\text { WP303 }\end{array}$ & 7 & 24 & 0.002 & Anxa8|Edn1|Ednrb|Pla2g4a|Ptger3|Ptgis|Ptgs1 \\
\hline Spinal cord injury: WP2433 & 19 & 92 & 0.025 & $\begin{array}{l}\text { Aqp4|Ccl2|Cxcl1|Epha4|Gap43|Gfap|Gja1| } \\
\text { LOC102550316ILtb|Mag|Ngfr|Pla2g2alPla2g5| } \\
\text { PrkcalRhoc|Selp|Sema6alVim|Xylt1 }\end{array}$ \\
\hline $\begin{array}{l}\text { GPCRs, class C metabotropic glutamate, } \\
\text { pheromone: WP42 }\end{array}$ & 5 & 15 & 0.008 & Gprc5alGprc5b|Gprc5clGrm4|Grm8 \\
\hline Keap1-Nrf2: WP1280 & 4 & 11 & 0.0075 & Gsta2IGsta5IMafINq01|Prkca \\
\hline Eicosanoid synthesis: WP293 & 5 & 16 & 0.006 & Alox5IPla2g2alPtges|Ptgis|Ptgs1 \\
\hline $\begin{array}{l}\text { Wnt signaling pathway and pluripotency: } \\
\text { WP1288 }\end{array}$ & 16 & 80 & 0.0375 & $\begin{array}{l}\text { Fzd1 ILef1INkd1 INkd2IPlaulPpm1j|Ppp2r2b|Prkcal } \\
\text { PrkcdIPrkcq|Tcf7IWnt10bIWnt11IWnt16IWnt2b| } \\
\text { Wnt6 }\end{array}$ \\
\hline Peptide GPCRs: WP131 & 11 & 50 & 0.048 & $\begin{array}{l}\text { Ccr1|Oprm1|Ednrb|Galr1|Npy1r|Npy5r|Oprk1| } \\
\text { Oprl1|Tacr3|Sstr4|Oprl }\end{array}$ \\
\hline
\end{tabular}

ELE, eletriptan.

We performed a hierarchical cluster analysis and found that genes upregulated and downregulated by ELE were similarly affected by INDO. Of note, profiles of differently expressed genes in trigeminal ganglia were almost the same with the threshold of 1.4 and 3 (Fig. 1B). To corroborate this finding, we analyzed the array data by means of the Venn diagram that describes the degree of overlapping of transcriptomic changes between 2 different treatments. We found that with a threshold of 1.4, a large number of genes upregulated or downregulated by ELE were similarly affected by INDO (Fig. 1C). A high degree of overlapping was also found when the threshold was set at 3 (Fig. 1D). The low number of inversely modulated genes found with the threshold of 1.4 was annulled when the threshold was set at 3 (Figs. 1C and D), further suggesting a very high degree of coherence of gene reprogramming within the trigeminal ganglion.

\section{Table 2}

Gene pathways downregulated or upregulated in the trigeminal ganglion of rats chronically exposed to INDO.

\begin{tabular}{|c|c|c|c|c|}
\hline Gene-set name & No. of altered genes & Total no. of genes & $P$ & Gene symbols \\
\hline \multicolumn{5}{|l|}{ Pathways downregulated } \\
\hline Cell cycle: WP429 & 14 & 74 & 0.001 & $\begin{array}{l}\text { Bub1|Bub1b|Ccna2|Ccnb1|Cdc6|Cdk1|Cdk6| } \\
\text { Cdkn2alEspl1|Mcm4|Mcm5|Mcm6|Pkmyt1|Skp2 }\end{array}$ \\
\hline G1 to S cell cycle control: WP348 & 10 & 58 & 0.003 & $\begin{array}{l}\text { Ccnb1|Cdk1|Cdk6ICdkn2alCreb3111/Mcm4|Mcm5| } \\
\text { Mcm6IMyc|Myt1 }\end{array}$ \\
\hline Hedgehog signaling pathway: WP574 & 4 & 17 & 0.022 & Ccnb1|Cdk1|Gli2\|lhh \\
\hline GPCRs, class B secretin-like: WP378 & 4 & 18 & 0.039 & Cd97|Crhr1|Gcgr|Ghrhr \\
\hline Nuclear receptors: WP217 & 6 & 36 & 0.0235 & ArlEsr1|Nr0b1|Nr4a2IPgr|Rorc \\
\hline GPCRs, other: WP409 & 8 & 59 & 0.0385 & $\begin{array}{l}\text { Cckbr|Celsr3|Drd4|Ghrhr|Gnrhr|Gpr143|Grm1| } \\
\text { Rxfp1 }\end{array}$ \\
\hline \multicolumn{5}{|l|}{ Pathways upregulated } \\
\hline Eicosanoid synthesis: WP293 & 6 & 16 & 0 & Alox15|Alox5|Pla2g2alPtges|Ptgis|Ptgs1 \\
\hline $\begin{array}{l}\text { GPCRs, class C metabotropic glutamate, } \\
\text { pheromone: WP42 }\end{array}$ & 5 & 15 & 0.0035 & Gprc5alGprc5b|Gprc5c|Grm3|Grm8 \\
\hline $\begin{array}{l}\text { Prostaglandin synthesis and regulation: } \\
\text { WP303 }\end{array}$ & 6 & 24 & 0.002 & Anxa3|Anxa8|Ednrb|Pla2g4a|Ptgis|Ptgs1 \\
\hline Matrix metalloproteinases: WP278 & 6 & 24 & 0.0025 & Mmp13|Mmp14|Mmp15|Mmp19|Mmp2|Mmp23 \\
\hline Peptide GPCRs: WP131 & 10 & 50 & 0.001 & $\begin{array}{l}\text { Ccr1ICcr6|Ednrb|Galr1|Npy1r|Npy5r|Oprk110prl11 } \\
\text { Sstr4|Tac4 }\end{array}$ \\
\hline Spinal cord injury: WP2433 & 16 & 93 & 0.038 & $\begin{array}{l}\text { Aqp4|Ccl2|Fcgr2alGfap|Gja1/L0C102550316ILtb| } \\
\text { Mag|Ngfr|Pla2g2alPla2g5|PrkcalRhoc|Sema6al } \\
\text { Tnfsf13IVim }\end{array}$ \\
\hline
\end{tabular}




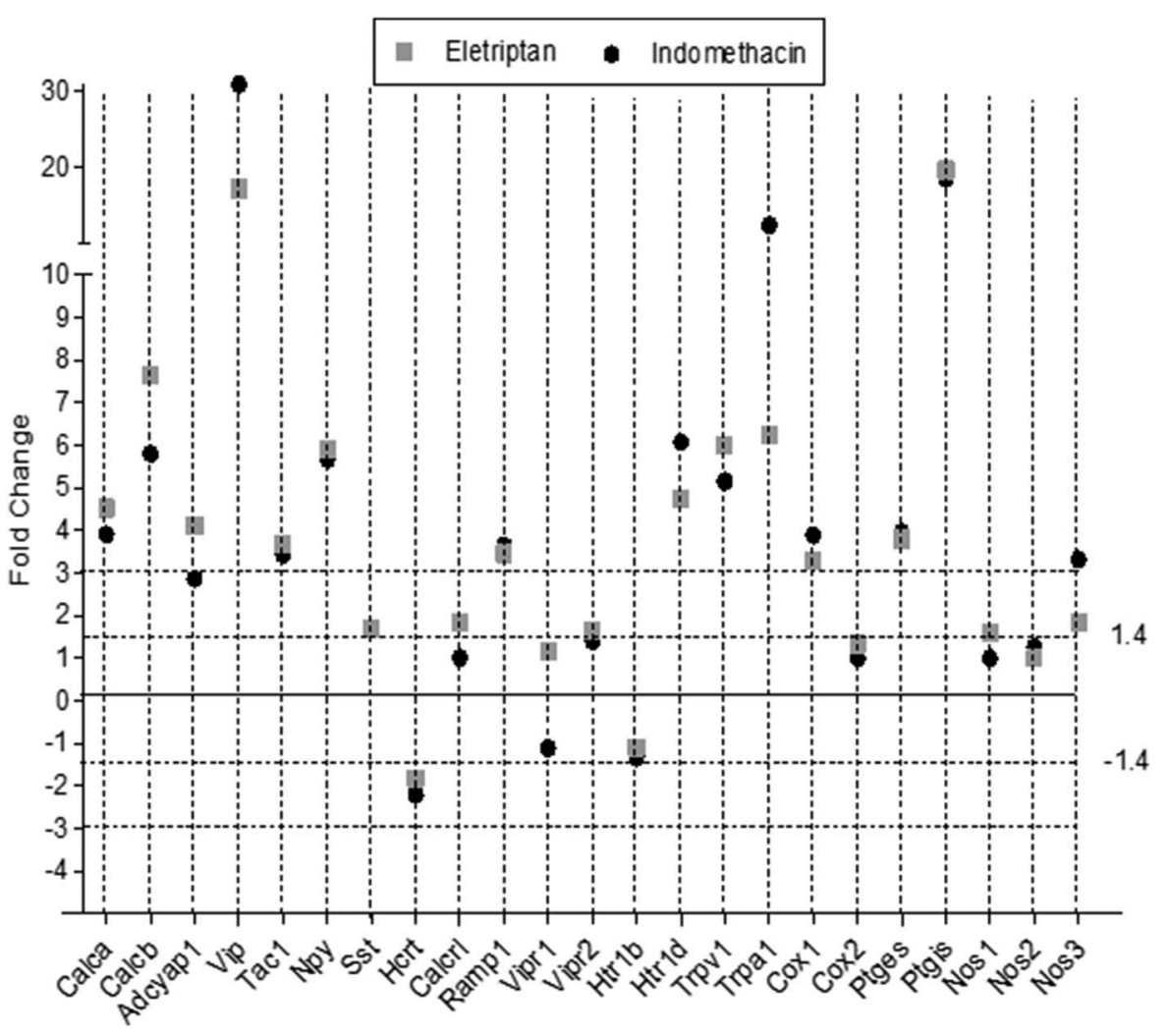

Figure 2. Transcriptomic analysis of specific headache-related genes in the trigeminal ganglion of rats exposed to chronic treatment with eletriptan (ELE) or indomethacin (INDO). The diagram shows the fold change of specific genes involved in headache pathogenesis selected from data obtained by the gene array analysis shown in Figure 1. The thresholds of 1.4 and 3 are shown as horizontal dashed lines. Calca, CGRP $\alpha$; Calcb, CGRP $\beta$; Adcyap1, PACAP; Vip, VIP; Tac1, substance P; Npy, NPY; Sst, somatostatin; Hcrt, hypocretin; Calcrl, calcitonin receptor-like; Ramp1, RAMP1; Vipr1, VIP receptor 1; Vipr2, VIP receptor 2; Htr1b, 5HT1B receptor; Htr1d, 5HT1D receptor; Trpv1, TRPV1 receptor; Trpa1, TRPA1 receptor; Ptgs1, COX1; Ptgs2, COX2; Ptges, PGE synthase; Ptgis, PGI synthase; Nos1, nNOS; Nos2, iNOS; Nos3, eNOS.

We then performed a pathway analysis of differently expressed genes adopting a threshold of 3. We found consistency among some of the most significantly altered pathways. Specifically, pathways downregulated by ELE and INDO were those involved in cell-cycle regulation and nuclear receptors, as well as Hedgehog signaling. Among the processes upregulated by both treatments, we found prostaglandin synthesis, genes associated with spinal cord injury, and signaling related to peptides acting on G-protein-coupled receptors (Tables 1 and 2).

The high correspondence of upregulated and downregulated genes by the 2 acute headache medications, prompted us to focus on the expression levels of specific genes reported to be of pathogenetic relevance to migraine and headache in general. Fold change of transcript levels of the single gene is reported in Figure 2. We found that genes coding for neuropeptides were all increased to a similar extend by ELE and INDO. Specifically, ELE and INDO increased CGRP $\alpha$ (Calca) transcripts by 4.52and 3.90-fold, respectively. Higher increases were found for CGRP $\beta$ (Calcb) transcripts that were augmented by 7.66- and 5.8-fold with ELE and INDO, respectively. PACAP (Adcyap1) transcripts augmented in the trigeminal ganglion of ELE- or INDO-treated rats by 4.11- and 2.87-fold, respectively. VIP (Vip) dramatically increased in ELE- and INDO-exposed animals by 17.15- and 30.88-fold, respectively. Similarly, substance P (Tac1) transcript levels were 3.68- and 3.43-fold higher than control in ELE- and INDO-treated rats, respectively. NPY (Npy) mRNA levels were augmented by ELE and INDO, showing a 5.91- and 5.67-fold increase, respectively. As for somatostatin
(Sst) transcripts, again we found that they were increased by ELE (1.70-fold) and INDO (1.68-fold). Similarly, transcripts for the hypocretin neuropeptide precursor (Hcrt, leading to the synthesis of orexins $A$ and $B$ ) appeared decreased by both ELE $(-2.2$-fold $)$ and INDO (-1.82-fold). Calcitonin receptor-like (Calcrl) transcripts were not changed by ELE, whereas appeared increased by INDO by 1.82-fold. Conversely, RAMP1 transcripts were increased by both ELE and INDO by 3.45- and 3.64-fold, respectively. Expression levels of 5HT1BR (Htr1b) appeared slightly decreased. Conversely, 5TH1DR (Htr1d) levels showed increases of 6.07- and 4.72-fold with ELE and INDO, respectively. Intriguingly, transcription of the key headache pain transducers [TRPV1 and TRPA1 receptors ${ }^{4}$ ] was increased. COX1 and COX2 appeared differently affected by the treatments. COX1 transcripts were increased by both ELE (3.89-fold) and INDO (3.28-fold), whereas those of COX2 did not show substantial changes. Of note, mRNAs for prostaglandin synthases that lay immediately downstream from COX and are responsible for the synthesis of effector vasodilating prostanoids were also increased by the 2 drugs. In particular, PGE2 synthase (Ptges) increased by 3.79- (ELE) and 3.97-fold (INDO), whereas a more pronounced increase of PGI2 synthase (Ptgis) was found with ELE (18.5-fold) and INDO (19.56-fold). Finally, eNOS (Nos3) transcript levels increased both with ELE (3.31fold) and INDO (1.83-fold) treatments; those coding for nNOS (Nos1) appeared slightly increased only by INDO (1.59-fold), whereas those coding for iNOS (Nos2) did not reach the threshold of 1.4 with both drugs (Fig. 2). 

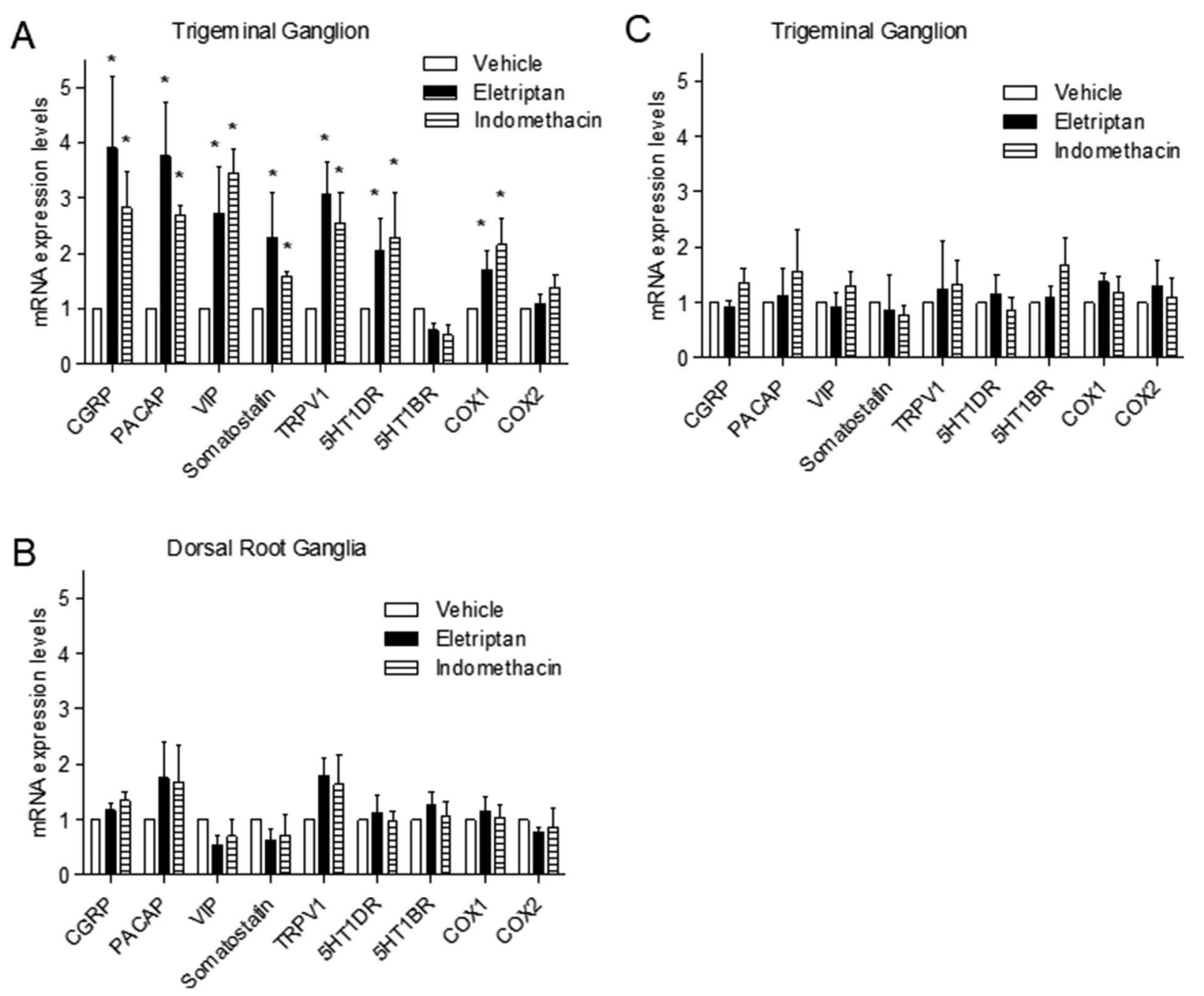

Figure 3. Real-time PCR confirmation of gene array data. Transcript levels of headache-related genes selected from Figure 2 have been quantified by means of qPCR in the trigeminal ganglion (A) or thoracic DRG (B) of rats exposed to ELE or INDO and compared with control animals. (C) qPCR in the trigeminal ganglion 10 days after abrupt interruption of the 1 -month treatment with ELE or INDO. Each column represents the mean $\pm \mathrm{SEM}$ of 10 rats. ${ }^{\star} P<0.05 \mathrm{vs}$ control, the Student $t$ test. CGRP, calcitonin gene-related peptide; DRG, dorsal root ganglion; ELE, eletriptan; INDO, indomethacin; PCR, polymerase chain reaction.

\subsection{Real-time polymerase chain reaction confirmation of hit gene transcripts identified by gene array analysis}

To corroborate our findings, we attempted to confirm the gene array data by means of qPCR analysis of genes that showed substantial expression changes. We therefore selected neuropeptides (CGRP $\alpha$, PACAP, VIP, and somatostatin), TRP channels (TRPV1), cyclooxygenases (COX1 and COX2) as well as serotonin receptors (5HT1BR and $5 \mathrm{HT} 1 \mathrm{DR})$. We extended this qPCR analysis to the cervico-thoracic DRG to understand whether gene expression changes induced by ELE and INDO were restricted to the trigeminal ganglion.

As shown in Figure 3A, qPCR fully confirmed increased expression of those genes identified by gene array analysis. In keeping with gene array data, gene expression of 5TH1BR and COX2 was not increased when evaluated by means of qPCR. Remarkably, when transcript levels of genes found increased in trigeminal ganglion were evaluated in thoracic DRG, only PACAP and TRPV1 showed a tendency toward increase albeit without reaching statistical significance (Fig. $3 \mathrm{~B}$ ). To mimic the clinical condition that occurs in patients with $\mathrm{MOH}$ once they undergo the prototypical 1-week detoxification protocol, ${ }^{3,11}$ we analyzed gene expression profiles in the trigeminal ganglion of rats 10 days after abrupt interruption of the 1-month treatment. We found that on interruption, a complete recovery of gene expression homeostasis occurs (Fig. 3C).

\subsection{Evaluation of pain thresholds in rats chronically exposed to ELE or INDO}

We next wondered whether the pronociceptive transcriptome alterations within the trigeminal ganglion of rats chronically exposed to ELE or INDO correlated with a functional state of orofacial allodynia and/or hyperalgesia. Alteration in pain thresholds was also analyzed in the forepaw in light of lack of transcriptional changes in the cervico-thoracic DRG. Interestingly, we found that orofacial allodynia evaluated by measuring tactile thresholds occurred in INDO- but not in ELE-exposed rats (Fig. 4A). Conversely, a pain test conducted with a low concentration of formalin showed that rats chronically treated with ELE but not INDO were hyperalgesic displaying vigorous orofacial rubbing behavior both during the early and delayed phase after formalin injection (Fig. 4B). To corroborate this finding, we also conducted a facial grimace test ${ }^{25}$ and found that only rats receiving ELE showed impressive and prolonged facial 
A

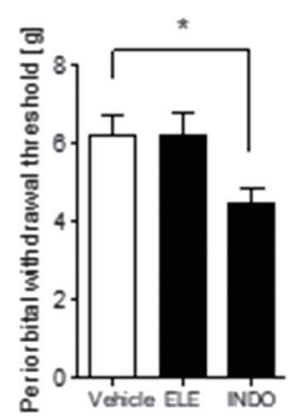

B

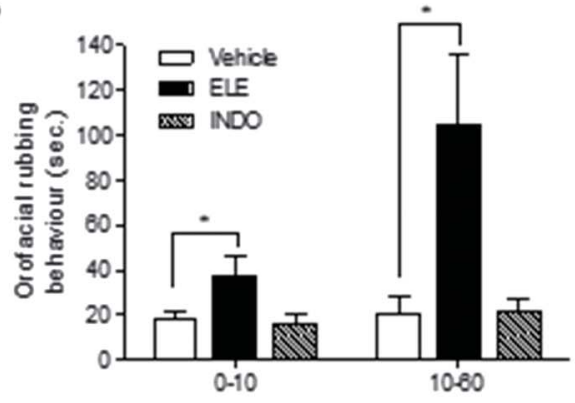

C

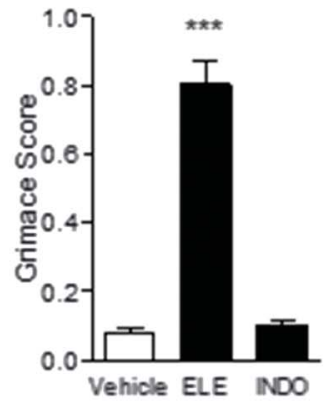

D

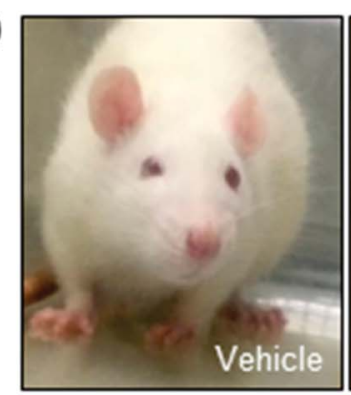

F

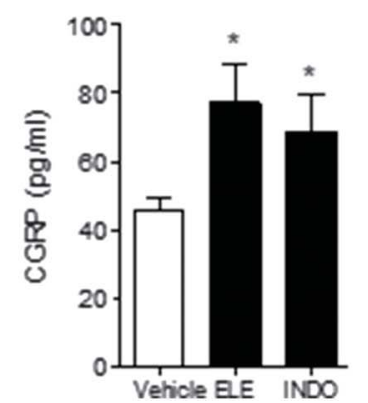

G

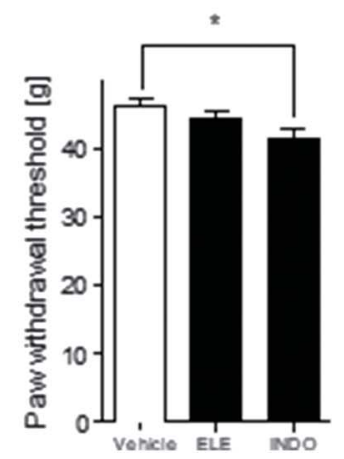

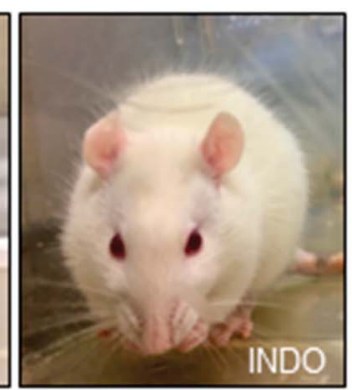

E

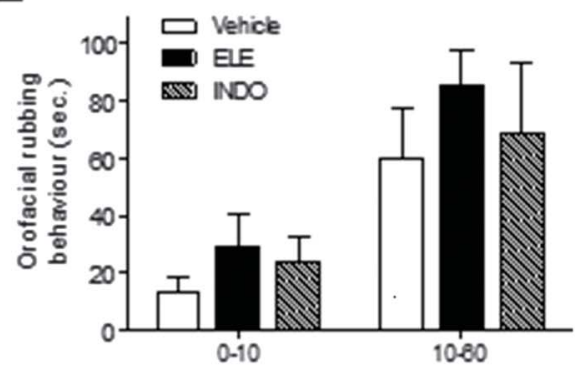

Figure 4. Sensory thresholds in rats chronically exposed to ELE or INDO. (A) Tactile withdrawal thresholds (the Von Frey test) and (B) rubbing behaviour (0.2\% periorbital formalin) in rats exposed for 1 month to daily oral administrations of vehicle, ELE or INDO. (C) Grimace score in rats injected with $0.2 \%$ periorbital formalin. (D) Representative images of rats during the grimace score. Note the obvious grimace in the animal chronically exposed to ELE. (E) Rubbing behaviour ( $0.5 \%$ periorbital formalin) in rats exposed for 1 month to daily oral administrations of vehicle, ELE, or INDO. (F) CGRP plasma level in blood collected from the retroorbital venous plexus after a challenge with a subcutaneous capsaicin injection in the supraorbital region. Tactile (G) and cold (H) withdrawal thresholds of the forepaw in rats exposed to vehicle, ELE, or INDO. Each column represents the mean \pm SEM of 10 rats. ${ }^{*} P<0.05$, ${ }^{\star} P<0.01$ vs vehicle. ANOVA plus the Tukey post hoc test. ANOVA, analysis of variance; CGRP, calcitonin gene-related peptide; ELE, eletriptan; INDO, indomethacin.

grimace on periorbital formalin injection (Figs. 4 C and D). To rule out the hypothesis that lack of periorbital sensitization was due to the analgesic properties of INDO, we analyzed orofacial pain intensity in rats injected with formalin at the suprathreshold concentration of $0.5 \%$. However, as shown in Figure 4E, rats chronically exposed to INDO did not display reduced orofacial rubbing when challenged with this concentration of formalin. Evidence of altered facial nociception, along with that of increased CGRP expression within the trigeminal ganglia of rats exposed to chronic ELE or INDO treatment, prompted us to investigate whether release of CGRP was also altered in these animals. We therefore measured the peptide in blood collected from the retro-orbital venous plexus after a challenge with a subcutaneous capsaicin injection in the supraorbital region. In keeping with transcriptional data, plasma levels of CGRP were significantly higher in ELE- and INDO-treated rats compared with controls (Fig. 4F). Next, we analyzed nociception thresholds at the forepaws and found that, in keeping with data obtained in the orofacial region, tactile threshold was only reduced in rats exposed to INDO (Fig. 4G). However, both INDO- and ELEtreated rats showed cold allodynia when evaluated with the cold plate test (Fig. 4H).

\section{Discussion}

Medication-overuse headache represents the most frequent transformation of chronic headache, leading to a clinical condition that exposes patients to severe secondary drug effects and strengthens headache chronicization. 5,6,10 This study shows that chronic exposure to 2 different acute headache remedies such as a triptan or an NSAID dramatically alters gene expression homeostasis within the trigeminal ganglion, as well as pain thresholds in the periorbital and forepaw regions. Importantly, quantitative and qualitative derangement of gene expression profiles is highly similar for both drugs, despite their different mechanisms of action. To the best of our knowledge, this is the first evidence that on chronic exposure to 2 different symptomatic headache remedies, the trigeminal ganglion activates the same 
transcriptional program to increase sensitivity to nociceptive stimuli. Notably, our preclinical data are in keeping with the clinic of patients with $\mathrm{MOH}$ that, although overusing different types of acute medications, invariably present the same pattern of symptoms. ${ }^{5,6,10}$ Our findings are also in keeping with previous work showing that repetitive exposure of rats to sumatriptan prompts a condition of latent sensitization to nociceptive stimuli concomitant with increased CGRP expression within the trigeminal ganglia. ${ }^{18-20,24}$

Currently, the exact neuroanatomical pathways and molecular mechanisms participating to trigeminal ganglion transcriptional reprogramming on chronic ELE or INDO exposure are unknown. A simple interpretation might be that each drug acts on its respective pharmacodynamic target on trigeminal ganglion neurons to prompt different pathways that then converge to prompt the same epigenetic reprogramming. Possibly, persistent INDO-dependent inhibition of prostanoid synthesis within the dura and other cranial structures, as well as changes in meningeal/cerebral vessel dynamics due to chronic ELE can be also sensed by peripheral trigeminal afferents that then signal gene expression changes back to the ganglion. Even chronic targeting of COX and 5HT1DR within the trigeminal nucleus caudalis can be sensed by central trigeminal afferents and might concur to resetting of gene expression in the ganglion. In light of the high degree of similarity of the pattern of gene reprogramming induced by ELE and INDO, we reason that signals originating from chronic targeting of 5HT1DR and COX are integrated by higher brain structures such as periaqueductal gray, hypothalamus, thalamus, and cortex 6 that then reset, as a sort of homeostatic feedback, trigeminal ganglion transcriptome, and functioning. Regardless of the brain structures involved, it is worth noting that high degree of consistency of gene resetting triggered by the 2 drugs in the trigeminal ganglion is the first experimental hint that a common neuroanatomical/neurochemical pathway is shared by symptomatic headache medicaments to induce $\mathrm{MOH}$. Furthermore, given that clinical features of $\mathrm{MOH}$ due to triptans or NSAIDs are essentially the same, it is likely that at least some of the genes found reset in rats are indeed relevant to $\mathrm{MOH}$ pathogenesis in patients.

This study indicates that transcriptional reprogramming in the trigeminal ganglion prompts pain sensitization and is a key neurobiological substrate of $\mathrm{MOH}$. Accordingly, we found that chronic INDO or ELE exposure leads to a pronociceptive state of periorbital allodynia and hyperalgesia, in good keeping with previous work from the Porreca group. ${ }^{18,19,24}$ It is worth noting, however, that despite similar transcriptome alterations, pain thresholds were not consistently affected by INDO and ELE. Indeed, only INDO prompted facial tactile allodynia, whereas periorbital hyperalgesia was found only in rats exposed to ELE. Although we do not know the reason(s) of this apparent discrepancy, we argue that events of synaptic plasticity underlying both allodynia and hyperalgesia do not lead to a static condition, but change over time and with different temporal kinetics during chronic exposure to INDO or ELE. These considerations taken together allow to speculate that $\mathrm{MOH}$ originates from a homeostatic response of the nervous system to chronic exposure to drugs such as analgesics or triptans that, eventually, alters functioning of the trigeminovascular system. Conversely, it is more difficult to hypothesize that resetting of gene expression profiles occurred because rats showed a drug dependence/addiction substrate. ${ }^{9}$ Medication-overuse headache might be a sort of compensatory state of trigeminal nociception when the latter is constantly repressed by symptomatic headache medications.
From a neurophysiological perspective, reprogramming of the trigeminovascular system with induction of pronociceptive genes might be a feedback mechanism in an attempt to reestablish pain thresholds.

The high degree of genomic plasticity induced by ELE and INDO within the trigeminal ganglion is at odds with lack of gene reprogramming in thoracic DRG. Previous work, however, demonstrates that rats chronically exposed to sumatriptan show allodynia not only in the periorbital region but also in the hind paw. ${ }^{19}$ Consistently, we now report that those rats exposed to INDO or ELE and showing no apparent alterations of gene expression profiles within the DRG innervating the forepaw do develop cold hyperalgesia in the same region. A possible explanation for this apparent inconsistency might be that sensitization of paws is due to spinal and/or supraspinal mechanisms and not to events occurring at the DRG. Accordingly, reduced supraspinal antinociceptive control has been shown in patients with $\mathrm{MOH},{ }^{29}$ representing the functional correlate of the high degree of maladaptive neuronal plasticity that takes place within high brain structures during migraine chronification. ${ }^{7}$ These results suggest, in keeping with a very recent report, ${ }^{26}$ that the trigeminal ganglion shows a peculiar, stereotyped (ie, drug-type independent) homeostatic response to chronic symptomatic headache medications that render the trigeminovascular system unique among other nociceptive pathways. This interpretation is also in keeping with a study (and routine clinical practice) showing that among patients with regular use of analgesics including NSAIDs for rheumatologic disorders, none developed a pattern of spontaneous peripheral pain accompanied by compulsive medication consumption, reminiscent of a sort of "medication overuse rheumatic pain." Still, $8 \%$ of these rheumatologic patients on chronic analgesics developed $\mathrm{MOH}$, but, strikingly, they all suffered from primary headache. ${ }^{1}$ Similarly, patients on chronic opioids for ulcerative colitis develop $\mathrm{MOH}$ only if they are migraineurs. ${ }^{37}$ This clinical information, along with present data on gene reprogramming in the trigeminal ganglion of rats, suggests that patients with headache have a neurophysiological trait that predisposes them to develop headache once their trigeminovascular system is in a state of latent pronociceptive sensitization. Indeed, given that the rats we used were not "migraineurs" despite their widespread trigeminal ganglion reprogramming of gene expression profiles, we hypothesize that the neurogenomic changes leading to pronociceptive sensitization might in large part occur in all the individuals exposed to repetitive analgesic intake. However, when they occur in a subject susceptible to headache, the specific neurophysiological substrate suffices to turn the latent pronociceptive condition into an overt $\mathrm{MOH}$.

In conclusion, this study furthers our understanding of the complex pathophysiology of the trigeminovascular system in condition of chronic exposure to symptomatic headache medications. Additional studies, however, are needed to understand whether pronociceptive gene resetting also occurs in the trigeminal ganglion of patients with $\mathrm{MOH}$, as well as to unravel neuroanatomy and neurochemistry underlying these genetic alterations and how they translate to headache.

\section{Conflict of interest statement}

The authors have no conflict of interest to declare.

This work was supported by grants from Italian Foundation for Multiple Sclerosis 2014/R/6 (recipient AC), Regione Toscana Rare Disease Projects-Heath Projects 2007 and 2009 (recipient 
AC), Italian Ministry of Health, Research Grant GR-201102352225, and ARISLA foundation.

\section{Article history:}

Received 19 March 2018

Received in revised form 10 May 2018

Accepted 14 May 2018

Available online 23 May 2018

\section{References}

[1] Bahra A, Walsh M, Menon S, Goadsby PJ. Does chronic daily headache arise de novo in association with regular use of analgesics? Headache 2003;43:179-90.

[2] Becker WJ. The diagnosis and management of chronic migraine in primary care. Headache 2017;57:1471-81.

[3] Bendtsen L, Munksgaard S, Tassorelli C, Nappi G, Katsarava Z, Lainez M, Leston J, Fadic R, Spadafora S, Stoppini A, Jensen R. Disability, anxiety and depression associated with medication-overuse headache can be considerably reduced by detoxification and prophylactic treatment. Results from a multicentre, multinational study (COMOESTAS project). Cephalalgia 2014;34:426-33.

[4] Benemei S, De Cesaris F, Fusi C, Rossi E, Lupi C, Geppetti P. TRPA1 and other TRP channels in migraine. J Headache Pain 2013;14:71.

[5] Bigal ME, Serrano D, Buse D, Scher A, Stewart WF, Lipton RB. Acute migraine medications and evolution from episodic to chronic migraine: a longitudinal population-based study. Headache 2008;48:1157-68.

[6] Bongsebandhu-phubhakdi S, Srikiatkhachorn A. Pathophysiology of medication-overuse headache: implications from animal studies. Curr Pain Headache Rep 2012;16:110-5.

[7] Brennan KC, Pietrobon D. A systems neuroscience approach to migraine. Neuron 2018;97:1004-21.

[8] Buonvicino D, Mazzola F, Zamporlini F, Resta F, Ranieri G, Camaioni E, Muzzi M, Zecchi R, Pieraccini G, Dolle C, Calamante M, Bartolucci G, Ziegler M, Stecca B, Raffaelli N, Chiarugi A. Identification of the nicotinamide salvage pathway as a new toxification route for antimetabolites. Cell Chem Biol 2018;25:471-82.

[9] Calabresi P, Cupini LM. Medication-overuse headache: similarities with drug addiction. Trends Pharmacol Sci 2005;26:62-8.

[10] Caproni S, Bianchi E, Cupini LM, Corbelli I, Beghi E, Calabresi P, Sarchielli $\mathrm{P}$. Long term headache duration is a factor predicting nonresponse to detoxification and advice in medication overuse headache. $\mathrm{J}$ Headache Pain 2014;15:88.

[11] Cevoli S, Giannini G, Favoni V, Terlizzi R, Sancisi E, Nicodemo M, Zanigni S, Bacchi Reggiani ML, Pierangeli G, Cortelli P. Treatment of withdrawal headache in patients with medication overuse headache: a pilot study. J Headache Pain 2017;18:56.

[12] Chaplan SR, Bach FW, Pogrel JW, Chung JM, Yaksh TL. Quantitative assessment of tactile allodynia in the rat paw. J Neurosci Methods 1994; 53:55-63.

[13] Colas R, Munoz P, Temprano R, Gomez C, Pascual J. Chronic daily headache with analgesic overuse: epidemiology and impact on quality of life. Neurology 2004;62:1338-42.

[14] Cupini LM, Calabresi P. Medication-overuse headache: pathophysiological insights. J Headache Pain 2005;6:199-202.

[15] D’Amico D, Grazzi L, Curone M, Leonardi M, Raggi A. Cost of medication overuse headache in Italian patients at the time-point of withdrawal: a retrospective study based on real data. Neurol Sci 2017;38:3-6.

[16] Da Silva AN, Lake AE III. Clinical aspects of medication overuse headaches. Headache 2014;54:211-7.

[17] De Felice M, Ossipov MH, Porreca F. Persistent medication-induced neural adaptations, descending facilitation, and medication overuse headache. Curr Opin Neurol 2011;24:193-6.
[18] De Felice M, Ossipov MH, Wang R, Dussor G, Lai J, Meng ID, Chichorro J, Andrews JS, Rakhit S, Maddaford S, Dodick D, Porreca F. Triptaninduced enhancement of neuronal nitric oxide synthase in trigeminal ganglion dural afferents underlies increased responsiveness to potential migraine triggers. Brain 2010;133:2475-88.

[19] De Felice M, Ossipov MH, Wang R, Lai J, Chichorro J, Meng I, Dodick DW, Vanderah TW, Dussor G, Porreca F. Triptan-induced latent sensitization: a possible basis for medication overuse headache. Ann Neurol 2010;67:325-37.

[20] De Felice M, Porreca F. Opiate-induced persistent pronociceptive trigeminal neural adaptations: potential relevance to opiate-induced medication overuse headache. Cephalalgia 2009;29:1277-84.

[21] Evers S, Marziniak M. Clinical features, pathophysiology, and treatment of medication-overuse headache. Lancet Neurol 2010;9:391-401.

[22] Fuh JL, Wang SJ. Dependent behavior in patients with medicationoveruse headache. Curr Pain Headache Rep 2012;16:73-9.

[23] Goadsby PJ. Is medication-overuse headache a distinct biological entity? Nat Clin Pract Neurol 2006;2:401.

[24] Hitomi S, Kross K, Kurose M, Porreca F, Meng ID. Activation of durasensitive trigeminal neurons and increased c-Fos protein induced by morphine withdrawal in the rostral ventromedial medulla. Cephalalgia 2017;37:407-17.

[25] Langford DJ, Bailey AL, Chanda ML, Clarke SE, Drummond TE, Echols S, Glick S, Ingrao J, Klassen-Ross T, Lacroix-Fralish ML, Matsumiya L, Sorge RE, Sotocinal SG, Tabaka JM, Wong D, van den Maagdenberg AM, Ferrari MD, Craig KD, Mogil JS. Coding of facial expressions of pain in the laboratory mouse. Nat Methods 2010;7:447-9.

[26] Levy D, Abdian L, Dekel-Steinkeller M, Defrin R. Experimental evidence for weaker endogenous inhibition of trigeminal pain than extra-trigeminal pain in healthy individuals. Cephalalgia 2018;38:1307-15.

[27] Luceri C, Bigagli E, Pitozzi V, Giovannelli L. A nutrigenomics approach for the study of anti-aging interventions: olive oil phenols and the modulation of gene and microRNA expression profiles in mouse brain. Eur J Nutr 2017;56:865-77.

[28] Meng ID, Dodick D, Ossipov MH, Porreca F. Pathophysiology of medication overuse headache: insights and hypotheses from preclinical studies. Cephalalgia 2011;31:851-60.

[29] Perrotta A, Serrao M, Sandrini G, Burstein R, Sances G, Rossi P, Bartolo M, Pierelli F, Nappi G. Sensitisation of spinal cord pain processing in medication overuse headache involves supraspinal pain control. Cephalalgia 2010;30:272-84.

[30] Probyn K, Bowers H, Caldwell F, Mistry D, Underwood M, Matharu M, Pincus T. Prognostic factors for chronic headache: a systematic review. Neurology 2017;89:291-301.

[31] Raboisson P, Dallel R. The orofacial formalin test. Neurosci Biobehav Rev 2004;28:219-26.

[32] Ren K. An improved method for assessing mechanical allodynia in the rat. Physiol Behav 1999;67:711-6.

[33] Russell MB, Lundqvist C. Prevention and management of medication overuse headache. Curr Opin Neurol 2012;25:290-5.

[34] Sakurai M, Egashira N, Kawashiri T, Yano T, Ikesue H, Oishi R. Oxaliplatin-induced neuropathy in the rat: involvement of oxalate in cold hyperalgesia but not mechanical allodynia. PAIN 2009;147:165-74.

[35] Sarchielli P, Corbelli I, Messina P, Cupini LM, Bernardi G, Bono G, Di Piero V, Petolicchio B, Livrea P, Prudenzano MP, Pini LA, Sandrini G, Allena M, Tedeschi G, Russo A, Caproni S, Beghi E, Calabresi P. Psychopathological comorbidities in medication-overuse headache: a multicentre clinical study. Eur J Neurol 2016;23:85-91.

[36] Tassorelli C, Jensen R, Allena M, De Icco R, Sances G, Katsarava Z, Lainez M, Leston J, Fadic R, Spadafora S, Pagani M, Nappi G. A consensus protocol for the management of medication-overuse headache: evaluation in a multicentric, multinational study. Cephalalgia 2014;34:645-55.

[37] Wilkinson SM, Becker WJ, Heine JA. Opiate use to control bowel motility may induce chronic daily headache in patients with migraine. Headache 2001;41:303-9. 